\title{
P-0786: Association of adiponectin with metabolic syndrome and its parameters among postmenopausal women of West Bengal, India
}

\author{
S. Srimani ${ }^{1}$, I. Saha ${ }^{2}$, D. Chaudhuri ${ }^{1}$ \\ ${ }^{1}$ All India Institute of Hygiene \& Public Health, Biochemistry \& Nutrition, Salt Lake, India. \\ ${ }^{2}$ IQ City Medical College and Narayana Multispeciality Hospital, Community Medicine, Durgapur, India.
}

\section{Background:}

Adiponectin, an essential adipocytokine imparts a key role in the development of metabolic syndrome (MS), a cluster of central adiposity and cardiovascular risk factors. Increased prevalence of MS after menopause has been reported.

\section{Objective:}

To find out the associations between serum adiponectin level and MS, as well as its different parameters among postmenopausal women in a rural area of West Bengal.

\section{Results:}

Table 1: Distribution of the subjects according to Adiponectin status in relation with metabolic syndrome, waist circumference, fasting blood glucose, triglyceride, HDL cholesterol, and blood pressure

$$
\mathbf{N}=\mathbf{1 7 0}
$$

\begin{tabular}{|c|c|c|c|c|}
\hline \multirow[b]{2}{*}{ Parameter } & \multicolumn{2}{|c|}{ Adiponectin level } & \multirow{2}{*}{$\begin{array}{c}\text { Total } \\
\text { Number } \\
(\%)\end{array}$} & \multirow{2}{*}{$\begin{array}{c}\text { Statistical } \\
\text { test - } \\
\text { Chi-square, } \\
\text { (P value) }\end{array}$} \\
\hline & $\begin{array}{l}<\text { Median } \\
(<9.72 \mu \mathrm{g} / \mathrm{ml})\end{array}$ & $\begin{array}{l}\geq \text { Median } \\
(\geq 9.72 \mu \mathrm{gg} / \mathrm{ml})\end{array}$ & & \\
\hline \multicolumn{5}{|c|}{ MS Present (IDF) } \\
\hline Yes & $59(62.76)$ & $35(37.24)$ & $94(100)$ & \multirow{2}{*}{$\begin{array}{c}13.7066 \\
(0.00024)\end{array}$} \\
\hline No & $26(34.21)$ & $50(65.79)$ & $76(100)$ & \\
\hline \multicolumn{5}{|c|}{ Waist circumference } \\
\hline$\geq 80 \mathrm{~cm}$ & $82(54.67)$ & $68(45.33)$ & $150(100)$ & \multirow{2}{*}{$\begin{array}{c}11.1067 \\
(0.00086)\end{array}$} \\
\hline$<80 \mathrm{~cm}$ & $03(15.0)$ & $17(85.0)$ & $20(100)$ & \\
\hline \multicolumn{5}{|l|}{ Fasting glucose } \\
\hline$\geq 100 \mathrm{mg} / \mathrm{dl}$ & $19(54.29)$ & $16(45.71)$ & $35(100)$ & \multirow{2}{*}{$\begin{array}{l}0.324 \\
(0.57) \\
\end{array}$} \\
\hline$<100 \mathrm{mg} / \mathrm{dl}$ & $66(48.89)$ & $69(51.11)$ & $135(100)$ & \\
\hline \multicolumn{5}{|l|}{ Triglyceride } \\
\hline$\geq 150 \mathrm{mg} / \mathrm{dl}$ & $36(53.73)$ & $31(46.27)$ & $67(100)$ & \multirow{2}{*}{$\begin{array}{c}0.6159 \\
(0.4326)\end{array}$} \\
\hline$<150 \mathrm{mg} / \mathrm{dl}$ & $49(47.57)$ & $54(52.43)$ & $103(100)$ & \\
\hline \multicolumn{5}{|c|}{ HDL cholesterol } \\
\hline$<50 \mathrm{mg} / \mathrm{dl}$ & $38(58.46)$ & $27(41.54)$ & $65(100)$ & \multirow{2}{*}{$\begin{array}{l}3.0139 \\
(0.082) \\
\end{array}$} \\
\hline$\geq 50 \mathrm{mg} / \mathrm{dl}$ & $47(44.76)$ & $58(55.24)$ & $105(100)$ & \\
\hline \multicolumn{5}{|l|}{ Blood Pressure } \\
\hline $\begin{array}{c}\geq 130 / 85 \mathrm{~mm} \text { of } \\
\mathrm{Hg}\end{array}$ & $66(60.0)$ & $44(40.0)$ & $110(100)$ & \multirow{2}{*}{$\begin{array}{c}12.4667 \\
(0.000414)\end{array}$} \\
\hline $\begin{array}{c}<130 / 85 \mathrm{~mm} \text { of } \\
\mathrm{Hg}\end{array}$ & 19 (31.67) & $41(68.33)$ & $60(100)$ & \\
\hline
\end{tabular}

* Statistically significant

\section{Conclusion:}

* Prevalence of metabolic syndrome: $55.3 \%$.

* Median adiponectin level: $9.72 \mu \mathrm{g} / \mathrm{ml}(1.03$ to $92.2 \mu \mathrm{g} / \mathrm{ml})$.

* Adiponectin was found to be associated with MS, central adiposity and hypertension among postmenopausal women of Singur block of West Bengal, India.

* Women with central adiposity, hyperglycaemia, hypertriglyceridaemia, hypertension and low HDL-C had significantly lower adiponectin level than their counterparts.

- Adiponectin level increased significantly with increase in HDL-C and decrease in SBP and DBP among the participants when taken together.

Receipt of grants or research support: Indian Council of Medical Research Junior Research Fellowship

Method:

$>$ Study Design : Community based Cross-sectional study.

> Study Area : 30 villages of Singur block, field practice area of All India Institute of Hygiene and Public Health (AllH\&PH), West Bengal, India.

> Study Population : 170 postmenopausal women aged 43 to 70 years; selected randomly

> Study period: June'2016 to September'2016.

$>$ Ethical Clearance: Ethics Committee of AllH\&PH.

Obtained written consent from all the participants before data collection.

$>$ Study Variables : Serum adiponectin, WC, FBG, TG, HDL-C and BP.

> Sample Collection : 8 to 10 hours fasting blood sample

$>$ Analysis:

* MS was defined as per International Diabetes Federation (IDF), 2005 (for Asian Indians) criteria.

* In absence of any cut off for serum adiponectin level, the study subjects were divided into two groups based on the observed median value of serum adiponectin level

* Statistical analysis - chi-square test $(x 2)$, Mann Whitney $U$ test $(Z$ value) and spearman's correlation coefficient (rho): SPSS software, version 20.0.

* P <0.05: Statistically significant.

Table 2: Relationship between waist circumference, fasting blood glucose, triglyceride, HDL cholesterol and blood pressure with serum Adiponectin level

\begin{tabular}{|c|c|c|c|}
\hline \multirow[b]{2}{*}{ Parameter } & \multirow[b]{2}{*}{$\begin{array}{l}\text { Adiponectin } \\
\text { level } \\
\text { Median (IQR) }\end{array}$} & \multicolumn{2}{|c|}{ Statistical test } \\
\hline & & $\begin{array}{c}\text { Mann Whitney } \\
U \text { test } \\
(P \text { value })\end{array}$ & $\begin{array}{c}\text { Spearman's } \\
\text { correlation } \\
\text { coefficient } \\
\text { (P value) }\end{array}$ \\
\hline \multicolumn{4}{|c|}{ Waist circumference } \\
\hline $\begin{array}{l}<80 \mathrm{~cm} \\
(\mathrm{n}=20)\end{array}$ & $\begin{array}{c}66.75 \\
(63-72.75)\end{array}$ & & \multirow{2}{*}{$\begin{array}{l}-0.14 \\
(0.06)\end{array}$} \\
\hline $\begin{array}{c}\geq 80 \mathrm{~cm} \\
(n=150)\end{array}$ & $\begin{array}{c}7.65 \\
(4.15-30.38)\end{array}$ & $\begin{array}{c}\mathrm{Z:}-15.16 \\
\mathrm{P:}<0.00001^{*}\end{array}$ & \\
\hline \multicolumn{4}{|c|}{ Fasting blood glucose } \\
\hline $\begin{array}{c}<100 \mathrm{mg} / \mathrm{dl} \\
(\mathrm{n}=135)\end{array}$ & $\begin{array}{c}10.57 \\
(4.65-39.67)\end{array}$ & \multirow{2}{*}{$\begin{array}{c}U: 1262 \\
Z:-14.55 \\
P:<0.00001^{*}\end{array}$} & \multirow{2}{*}{$\begin{array}{c}-0.15 \\
(0.055)\end{array}$} \\
\hline $\begin{array}{c}\geq 100 \mathrm{mg} / \mathrm{dl} \\
(\mathrm{n}=35)\end{array}$ & $\begin{array}{c}6.32 \\
(3.09-24.42)\end{array}$ & & \\
\hline \multicolumn{4}{|l|}{ Triglyceride } \\
\hline $\begin{array}{c}<150 \mathrm{mg} / \mathrm{dl} \\
(\mathrm{n}=103)\end{array}$ & $\begin{array}{c}10.77 \\
(4.34-31.44)\end{array}$ & \multirow{2}{*}{$\begin{array}{c}U: 375 \\
Z:-15.53 \\
P:<0.00001^{*}\end{array}$} & \multirow{2}{*}{$\begin{array}{l}-0.09 \\
(0.23)\end{array}$} \\
\hline $\begin{array}{c}\geq 150 \mathrm{mg} / \mathrm{dl} \\
(\mathrm{n}=67)\end{array}$ & $\begin{array}{c}9.04 \\
(3.89-39.67)\end{array}$ & & \\
\hline \multicolumn{4}{|c|}{ HDL cholesterol } \\
\hline $\begin{array}{c}<50 \mathrm{mg} / \mathrm{dl} \\
(\mathrm{n}=65)\end{array}$ & $\begin{array}{c}6.025 \\
(3.35-26.71)\end{array}$ & \multirow{2}{*}{$\begin{array}{c}\text { U: } 3900 \\
Z:-11.64 \\
P:<0.00001^{*}\end{array}$} & \multirow{2}{*}{$\begin{array}{c}0.18 \\
(0.01)^{*}\end{array}$} \\
\hline $\begin{array}{c}\geq 50 \mathrm{mg} / \mathrm{dl} \\
(\mathrm{n}=105)\end{array}$ & $\begin{array}{c}11.26 \\
(4.83-39.78)\end{array}$ & & \\
\hline \multicolumn{4}{|l|}{ Blood Pressure } \\
\hline $\begin{array}{c}\text { SBP }<130 \\
(n=63)\end{array}$ & $\begin{array}{c}14.74 \\
(5.42-48.84)\end{array}$ & \multirow{2}{*}{$\begin{array}{c}\mathrm{U}: 107 \\
\mathrm{Z}:-15.83 \\
\mathrm{P}:<0.00001^{*}\end{array}$} & \multirow{2}{*}{$\begin{array}{c}-0.17 \\
(0.02)\end{array}$} \\
\hline $\begin{array}{c}\text { SBP } \geq 130 \\
(n=107)\end{array}$ & $\begin{array}{c}7.18 \\
(4.0-24.42) \\
\end{array}$ & & \\
\hline $\begin{array}{c}\text { DBP }<85 \\
(n=92)\end{array}$ & $\begin{array}{c}11.29 \\
(5.13-36.93)\end{array}$ & \multirow{2}{*}{$\begin{array}{c}U: 1021 \\
Z:-14.82 \\
P:<0.00001^{*}\end{array}$} & \multirow{2}{*}{$\begin{array}{c}-0.19 \\
(0.01)^{*}\end{array}$} \\
\hline $\begin{array}{c}\text { DBP } \geq 85 \\
(n=78)\end{array}$ & $\begin{array}{c}7.60 \\
(3.91-35.63)\end{array}$ & & \\
\hline
\end{tabular}

